\title{
Corrección quirúrgica de la incontinencia de orina de esfuerzo, con cinta sintética sub-medio-uretral de tercera generación: TVT-Secur
}

\author{
Solà Dalenz V, Ricci Arriola P.*, Pardo Schanz. J. \\ Unidad de Uroginecología y Cirugía Vaginal, Clinica Las Condes, Santiago (Chile).
}

Actas Urol Esp. 2008;32(5):522-529

\section{RESUMEN}

\section{CORRECCIÓN QUIRÚRGICA DE LA INCONTINENCIA DE ORINA DE ESFUERZO, CON CINTA SINTÉTICA} SUB-MEDIO-URETRAL DE TERCERA GENERACIÓN: TVT-SECUR

Objetivo: Revisar nuestra experiencia inicial en la corrección de la incontinencia de orina de esfuerzo, con el nuevo sistema TVT-Secur. Revisar la efectividad y seguridad de esta técnica.

Método: Entre enero y mayo del 2007, se realizaron 16 TVT-Secur (Women's Health \& Urology, Ethicon, Johnson \& Johnson) a pacientes de la Unidad de Uroginecología y Cirugía Vaginal, del Departamento de Ginecología y Obstetricia de la Clínica Las Condes. En 6 pacientes la cinta se situó en "V" y en 10 en "U". La mediana de edad para el grupo fue de 52 años, índice de masa corporal 29, paridad 3. En todas se realizó estudio urodinámico previo como parte del estudio y selección preoperatoria. Todas debían tener al menos un año de incontinencia de orina de esfuerzo, y ninguna debía tener corrección quirúrgica previa.

Resultado: La mediana de tiempo operatorio fue de 10 minutos, con un rango entre 8 y 15 minutos. No se presentaron complicaciones durante el acto operatorio, ni en el postoperatorio inmediato, temprano y tardío. La EVA registrada a las 12 horas fue de 1 a 2. Se obtuvo corrección de la incontinencia de orina de esfuerzo en todas las pacientes. El tiempo de seguimiento fue entre 1 y 4 meses, con una mediana de 2 meses. Todas expresaron estar conformes con los resultados de la cirugía y la recomendarían a otras pacientes en igual situación.

Conclusiones: Según nuestra experiencia inicial, el nuevo sistema TVT-Secur, aparece como eficaz y seguro, en la quirúrgica de la incontinencia de orina de esfuerzo. Sin embargo, sólo el seguimiento a largo plazo, y la incorporación de nuevos pacientes al estudio, permitirá determinar el mantenimiento de estos buenos resultados en el tiempo.

Palabras clave: TVT-Secur. Cinta sub-medio-uretral. Incontinencia de orina de esfuerzo. Cirugía mínimamente invasiva.

\section{ABSTRACT \\ STRESS URINARY INCONTINENCE SURGICAL CORRECTION WITH THIRD GENERATION SUB-MID-URETHRA SLING: TVT-SECUR}

Objective: To review our initial experience with the new system TVT-Secur for the stress urinary incontinence. To evaluate the feasibility, efficacy and main advantages of the surgical correction with the new device.

Method: Between january and may of 2007, 16 TVT-Secur (Women's Health \& Urology, Ethicon, Johnson \& Johnson) were made in patients of the Urogynecology Unit, of Clínica Las Condes. Six TVT-Secur were applied in "V" and 10 in "U". The median age was 52 years old, BMI 29, parity of 3. All patients were studied with urodynamic before surgery. All must have the urinary incontinence by more of one year and no must have previous surgical correction.

Results: The media surgical time was 10 minutes (8-15 minutes). Complications were not registered during intraoperative and immediate, early or delayed postoperative time. The visual analogue scale of pain was 1 to 2 at 12 hours. All patients were cured. The follow-up was between 1 and 4 months, 2 month media. All patients expressed satisfaction with the surgical results and they would recommend it to other patients in the same conditions.

Conclusion: According to our initial experience, the new system TVT-Secur is feasible, safe and effective in the surgical correction of the SUI. However, only the long follow-up and the incorporation of new patients to the study, will allow to determine the permanence of these good results in the time.

Keywords: TVT-Secur. Sub-mid-urethral Sling. Stress urinary incontinence. Minimal invasive surgery. 
$\mathrm{L}^{2}$ a incontinencia de orina de esfuerzo es una patología de alta prevalencia, que al igual que otros trastornos del suelo pélvico, como el prolapso, aumenta progresivamente con la edad y la paridad ${ }^{1}$. Se estima que 1 de cada 4 mujeres adultas es portadora de incontinencia urinaria ${ }^{2}$. Se trata de una patología que afecta con un alto grado de impacto a la calidad de vida, ya que reduce la autonomía y afecta la autoestima de quien la padece. Por estas razones, numerosos han sido los esfuerzos por desarrollar diferentes cirugías para su corrección, sin embargo, una de las más exitosas han sido las que sitúan una cinta en posición sub-medio-uretral. Estas técnicas quirúrgicas son sustentadas por la teoría de la hamaca, desarrollada por De Lancey y luego Petros y Ulmsten. Se trata de técnicas quirúrgicas que ofrecen todas las ventajas de una cirugía mínimamente invasiva con un alto porcentaje de cura. En 1996 Ulmsten presentó la primera de ellas, el TVT (Tension-Free-Vaginal-Tape) ${ }^{3,4}$. Se trata de una técnica con cifras de hasta $81 \%$ de curación completa y $16 \%$ parcial en seguimiento a 7 años ${ }^{5}$. Esta técnica requiere de cistoscopia para comprobar la indemnidad vesical ${ }^{6}$. Durante el 2001 Delorme describe la técnica de TOT (Trans-ObturatorTape) $)^{7,8}$, técnica en que las agujas no pasan por el espacio retropúbico, reduciendo la posibilidad de perforación vesical, por lo que no requiere una cistoscopia de revisión ${ }^{9}$. En un intento por simplificar y hacer aún más segura la técnica, Jean de Leval, durante el 2003, describe el TVT-O (Trans-VaginalTape-Obturator) ${ }^{10,11}$.
Cuando creíamos que era imposible una mayor simplificación, durante el último trimestre del 2006 se presentó una nueva técnica, el TVT-Secur ${ }^{12}$. Técnica que permite colocar una cinta sub-mediouretral en "U", como en el TVT o en "V" como en el TOT.

Con el objetivo de revisar la factibilidad, seguridad y eficacia del nuevo sistema TVT-Secur, analizamos en forma prospectiva nuestra experiencia inicial con esta nueva técnica.

\section{PACIENTE Y MÉTODO}

Protocolo de Estudio: Seguimiento prospectivo de 16 pacientes ingresadas a la Unidad de Uroginecología y Cirugía Vaginal de Clínica Las Condes, para corrección de la IOE por medio del nuevo sistema de aplicación de cinta sub-medio-uretral, TVTSecur (Women's Health \& Urology, Ethicon, Johnson \& Johnson), entre enero y mayo del 2007.

Criterio de inclusión: Todas las pacientes ingresadas al estudio debían presentar incontinencia de orina de esfuerzo al menos por un año y de carácter permanente. No debían tener antecedente de tratamiento previo por esta causa. A todas se les realizó una urodinamia monocanal con medición de retro-resistencia uretral y cistometrograma ${ }^{13,14}$. En todas se confirmó una hipermobilidad uretral por medio de un $\mathrm{Q}$-Tip test.

Caracteristicas del grupo de pacientes ingresadas al estudio: La media de edad fue de 52 años, el índice de masa corporal de 29 y la paridad de 3 . (Tablas 1 y 2).

Tabla 1. Casos ingresados al estudio

\begin{tabular}{|c|c|c|c|c|c|}
\hline Caso & Edad & Urodinamia Monocanal & TVT-S (Tipo) & Cistoscopia & EVA 12 Hrs \\
\hline 1 & 52 & II+III & $\mathrm{U}$ & SÍ & 2 \\
\hline 2 & 72 & II & $\mathrm{V}$ & NO & 1 \\
\hline 3 & 52 & II+III & $\mathrm{U}$ & SÍ & 1 \\
\hline 4 & 46 & II+III & $\mathrm{U}$ & Sí & 1 \\
\hline 5 & 46 & II+III & $\mathrm{U}$ & SÍ & 2 \\
\hline 6 & 52 & II & $\mathrm{V}$ & NO & 2 \\
\hline 7 & 42 & II+III & $\mathrm{U}$ & Sí & 2 \\
\hline 8 & 31 & II & $\mathrm{V}$ & NO & 1 \\
\hline 9 & 50 & II+III & $\mathrm{U}$ & SÍ & 2 \\
\hline 10 & 53 & II & $\mathrm{V}$ & NO & 1 \\
\hline 11 & 60 & II & $\mathrm{V}$ & NO & 2 \\
\hline 12 & 52 & II & $\mathrm{V}$ & NO & 2 \\
\hline 13 & 54 & II+III & $\mathrm{U}$ & SÍ & 1 \\
\hline 14 & 56 & $\mathrm{II}+\mathrm{III}$ & $\mathrm{U}$ & SÍ & 2 \\
\hline 15 & 59 & $\mathrm{II}+\mathrm{III}$ & $\mathrm{U}$ & SÍ & 2 \\
\hline 16 & 78 & II+III & $\mathrm{U}$ & SÍ & 2 \\
\hline
\end{tabular}


Tabla 2. Resumen del grupo ingresado a estudio.

\begin{tabular}{|c|c|c|c|}
\hline Caracteristicas Pacientes & Rango & & Media \\
\hline Edad & 31 a 59 años & & 52 años \\
\hline IMC & 25 a 37 & & 29 \\
\hline Paridad & 2 a 4 & & 3 \\
\hline Tiempo operatorio & 8 a 15 minutos & & 10 minutos \\
\hline \multicolumn{4}{|l|}{ Complicaciones } \\
\hline Intraoperatoria & & No se registraron & \\
\hline Postoperatorio Inmediato (hasta 24 h) & No se registraron & & \\
\hline Postoperatorio Temprano (hasta 7 dias) & No se registraron & & \\
\hline Postoperatorio Tardío & (después de 7 días) & No se registraron & \\
\hline EVA 12 h (sin cirugia asociada) & 1 a 2 & & \\
\hline Periodo de seguimiento & 1 a 4 meses & & media 2 meses \\
\hline
\end{tabular}

(EVA) Escala Visual Análoga del Dolor.

La urodinamia mostró incontinencia de esfuerzo tipo II en 6 pacientes y tipo II+III en 10 (Clasificación de la incontinencia de orina de esfuerzo de McGuire et al. $)^{15}$.

Criterio de elección de la posición de la cinta: En las mujeres con IOE tipo II+III, se colocó la cinta sub-medio-uretral en "U" y en las con tipo II en "V".

Tipo de anestesia: Se utilizó anestesia espinal.

Descripción del dispositivo TVT-Secur: El dispositivo está formado por dos ramas que en su extremo anterior soportan una malla de polipropileno macroporo monofilamento (idéntica a la de TVT clásico). La malla en el lugar de sujeción a las ramas, se encuentra a manera de sándwich encerrada por dos láminas de material de fijación absorbible, vicryl (poliglactin 910) y sutura de PDS (polidioxanon). En el otro extremo (posterior) posee un sistema que permite soltar la malla una vez puesta bajo la uretra media, a través de la tracción de una prolongación metálica. La rama derecha en su extremo proximal (el de la malla) posee una cubierta protectora que permite identificarla. (Fig. 1).

El dispositivo TVT-Secur permite colocar la malla bajo la uretra en "V" (como en el TOT o el TVTO) o en "U" (como en el TVT).

\section{Técnica quirúrgica específica:}

Paso 1: una vez anestesiada la paciente, se coloca en posición ginecológica en 90 grados de flexión muslo-abdomen. Se instala sonda Foley 16 French, permitiendo un vaciamiento completo de la vejiga.

Paso 2: se realiza una incisión longitudinal de 12 $\mathrm{mm}$ en la mucosa vaginal, a nivel de tercio medio uretral. (Figs. 2 y 3).

\section{Paso 3:}

A). Si se opta por la colocación en "U", se realiza una disección en 45 grados respecto a la línea media, con tijeras de Metzenbaun en el tejido parauretral. Esta disección es de 2 a 2,5 cm de longitud. (Fig. 4). Antes del siguiente paso, en este caso, se debe colocar un movilizador vesical dentro de una sonda Foley para desplazar la vejiga contra lateralmente al lado donde se inserta la rama del dispositivo.

B). Si se opta por colocación en "Hammock" (en "V") se realiza una disección en más de 45 grados (unos 67 grados), también de 2 a 2,5 cm de longitud (Fig. 4).

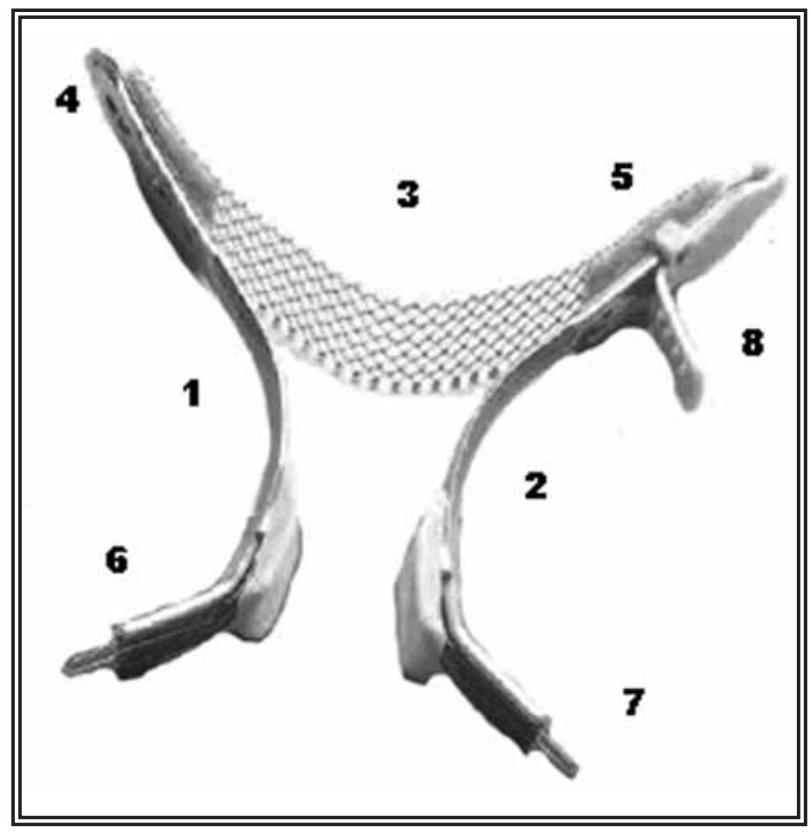

FIGURA 1. 


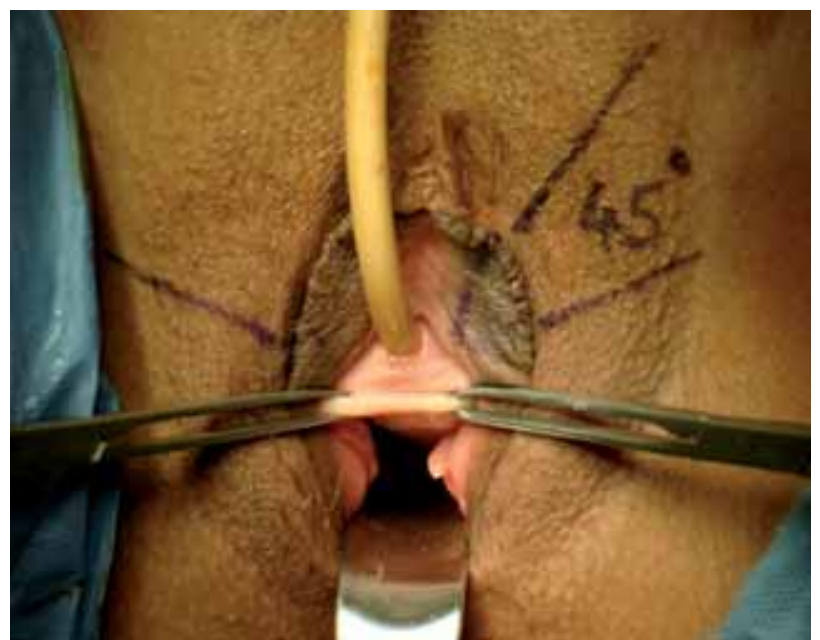

FIGURA 2.

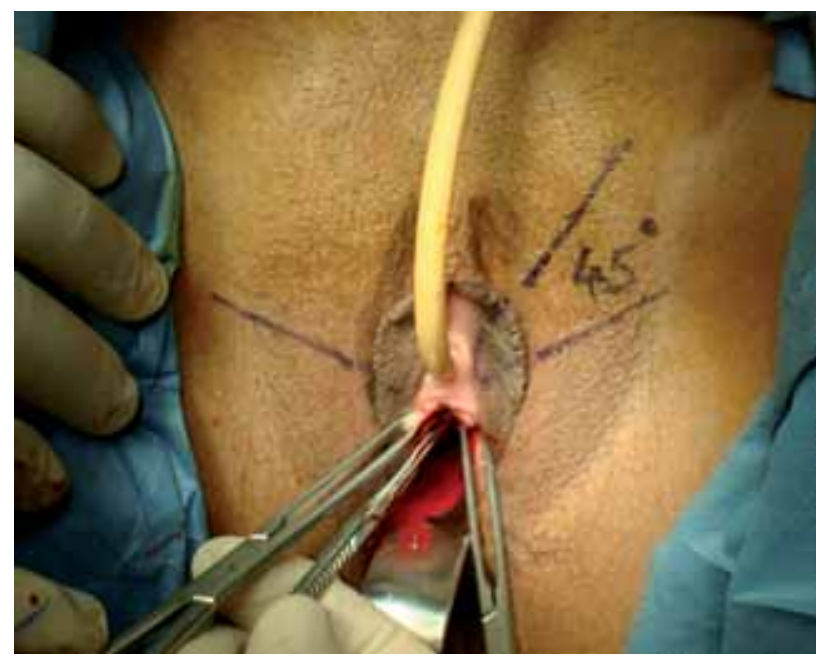

FIGURA 3.

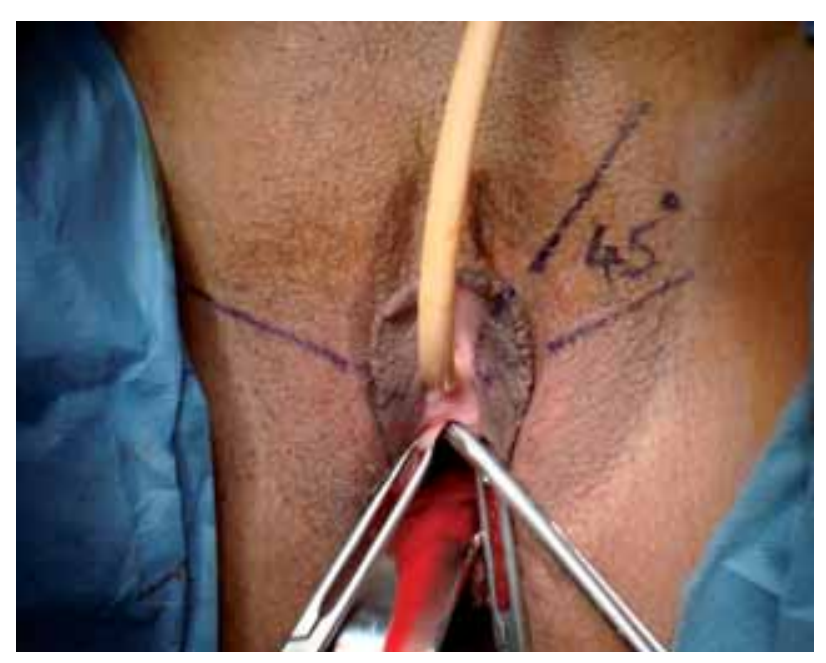

FIGURA 4.
Paso 4: Se toma la rama del dispositivo TVTSecur que se va a introducir, con un porta aguja, por su extremo distal, y se introduce en el espacio disecado (Fig. 5). Se termina de introducir mediante la presión manual (Fig. 6). Se repite la maniobra con la rama contra lateral, ajustando la cinta de polipropileno bajo la uretra media, siempre con técnica libre de tensión. (Fig. 7).

Si se ha optado por dejar la cinta en "U", se debe realizar una cistoscopia de revisión para comprobar indemnidad vesico-uretral, al igual que en la técnica de TVT.

Paso 5: Se retira el mango de inserción de cada lado con un pequeño movimiento de rotación y tracción, empujando el extremo de fijación del dispositi-

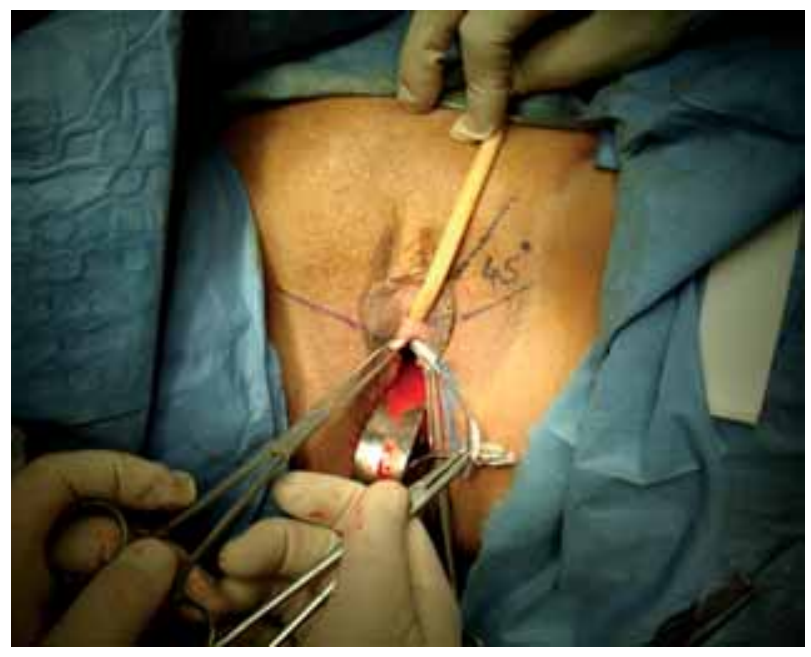

FIGURA 5.

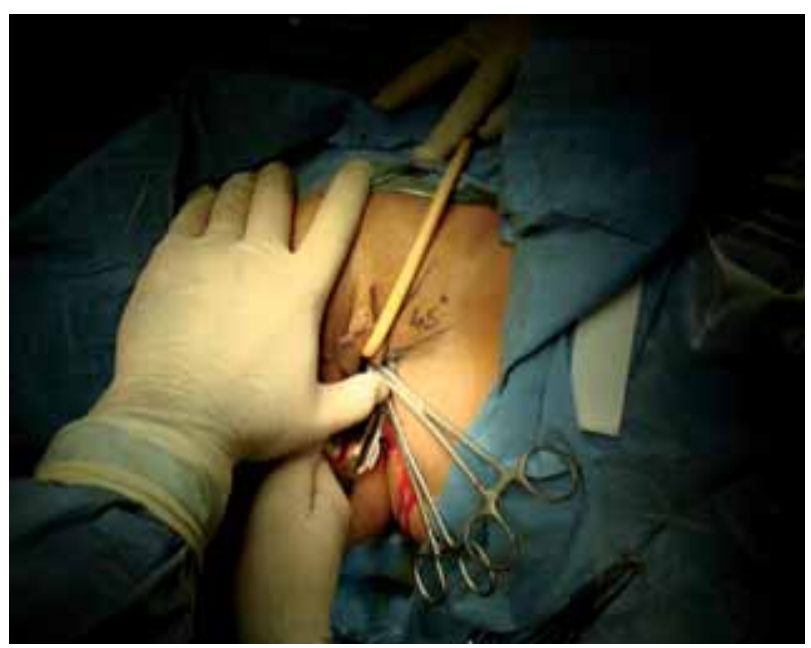

FIGURA 6. 


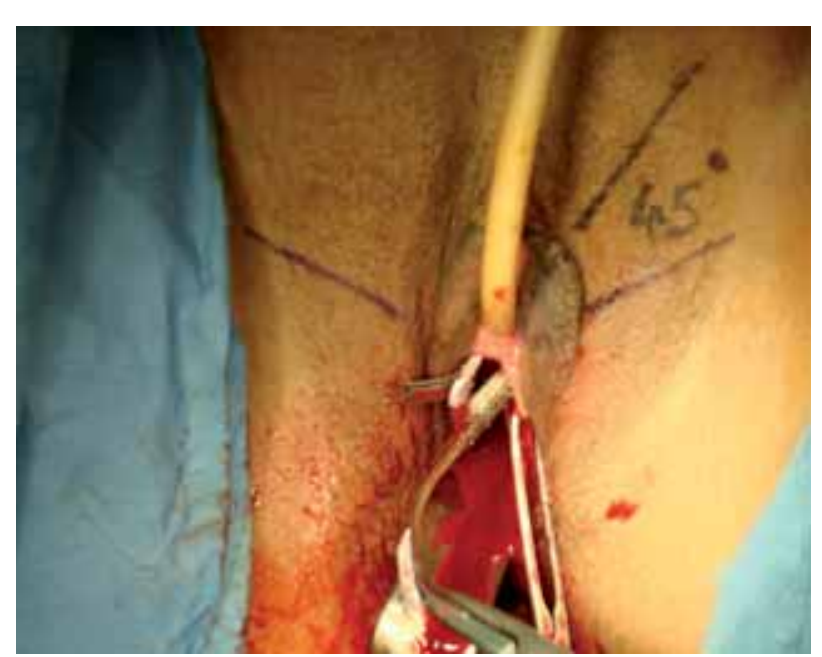

FIGURA 7.

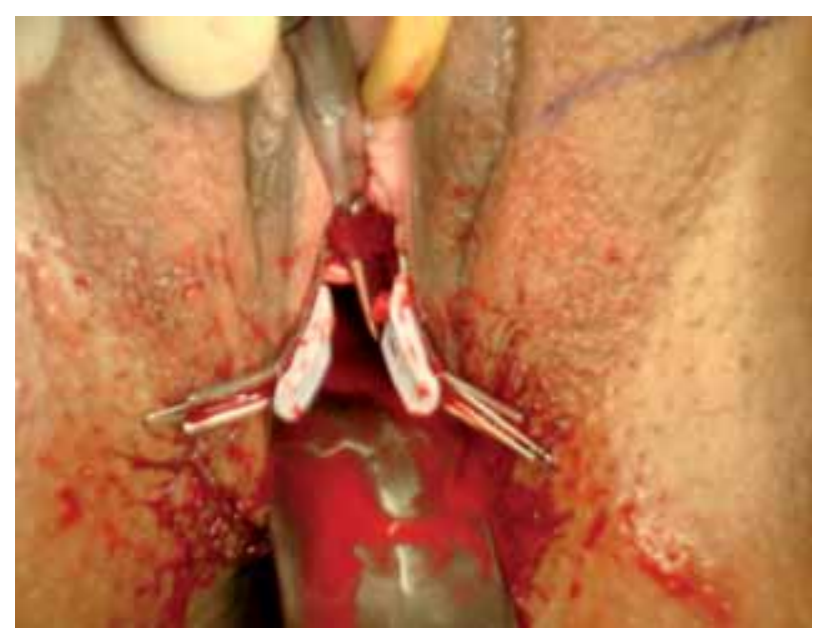

FIGURA 8.

vo hacia las estructuras óseas de la pelvis. En caso necesario, antes de retirar ambos mangos se puede volver a rectificar el ajuste, por medio de la tracción de las ramas y/o con una tijera de Metzembaum (Fig. 8).

Paso 6: se realiza una colporrafia con vicryl 3-0 corrido.

Si se opta por la colocación de la cinta suburetral en "Hammock" se puede prescindir del movilizador vesical y de la cistoscopia de revisión.

Retiro de Sonda: Se retiró 2 horas después de la cirugía.

Analgesia: Se utilizó ketoprofeno 100 mg endovenoso cada 8 horas, por tres dosis. Luego ibuprofeno $600 \mathrm{mg}$ cada 12 horas vía oral, por 7 días.
Levantada y Deambulación: A las 4 horas.

Alta hospitalaria: A las 12 horas, después de verificar dos micciones espontáneas.

Registro subjetivo del dolor: Mediante escala visual análoga del dolor (EVA) a las 12 horas de postoperatorio.

Control ambulatorio: A los siete y treinta días. Durante este control se preguntó el grado de satisfacción con la cirugía y los resultados obtenidos.

Criterio de curación, mejoría y fracaso: Se clasificó el resultado de la cirugía, según el número de episodios de incontinencia de orina registrados durante el período de observación. Se considera cura a la ausencia de incontinencia. Mejoría a la presencia de episodios de incontinencia menos que una vez cada dos semanas. Falla a la presencia de episodios de incontinencia más de una vez por semana.

\section{RESULTADOS}

El tiempo operatorio fluctuó entre 8 y 15 minutos, con una media de 10 minutos.

Se completó un período de observación entre 1 y 5 meses, con una media de 2 meses.

No se registraron complicaciones en el intraoperatorio, ni en postoperatorio inmediato (primeras 24 horas), temprano (primeros 7 días) ni tardío (después de los 7 días).

La escala visual análoga del dolor, aplicada a las 12 horas fue entre 1 y 2 . No siendo necesario agregar otros medicamentos al esquema previamente establecido.

En todas las pacientes se corrigió la IOE y todas refirieron estar conformes con la cirugía y sus resultados. Todas expresaron que recomendarían la técnica a otras pacientes en iguales circunstancias.

\section{DISCUSIÓN}

La eficacia de los sistemas de corrección con cinta sub-medio-uretral ya ha sido probada con la técnica de TVT. Este sistema constituyó la primera generación de cintas bajo la uretra para la corrección quirúrgica de la incontinencia de orina de esfuerzo. Todos los cambios posteriores pretendieron simplificar la técnica. Tal es el caso de las cintas de segunda generación, en que se evita la cistoscopia, al salir sus extremos por el agujero obturador (TOT y TVT-O). Estas cintas no pasan por el espacio retropúbico a diferencia del TVT. De esta 
forma disminuye la posibilidad de perforación vesical y de intestino, que son complicaciones descritas en la técnica de TVT $^{16,17}$. Una publicación reciente, realizada por nuestro grupo de trabajo, demostró que tanto el TVT como el TVT-O son técnicas efectivas en la corrección de la IOE, sin embargo, el TVTO es más fácil de realizar y posee menos posibilidad de complicaciones intraoperatorias ${ }^{17}$.

El TVT-Secur, representa una tercera generación de cintas, en que se ha simplificado aún más la técnica, al compararlo con sus antecesores. Este nuevo sistema se presenta con una versatilidad, sin precedentes, ya que permite colocar la cinta en "U" (como en el TVT) o en "V" (como en el TOT o TVT-O).

Distintos reportes de complicaciones en cirugías anti-incontinencia con cintas transobturadoras, aunque escasos, han informado casos de daño del nervio obturador, sangrado abundante (igual o mayor a $600 \mathrm{cc}$ ) y abscesos de la fosa isquiorectal ${ }^{18}$. Teóricamente, con el sistema TVT-Secur podrían reducirse estas complicaciones, ya que se trata de una cinta de menor longitud (8 centímetros), que no requiere una salida por la piel, sólo una pequeña incisión vaginal. Por lo que se trata de una técnica de mínima invasión, que al compararla con las anteriores, cumple de mejor manera este concepto.

Durante el 2006, Rezapour et al. ${ }^{19}$ presentaron la experiencia y evaluación preclínica de este nuevo sistema. Este estudio fue realizado en ovejas, y midió la mantención de la fuerza de tensión bajo la uretra, a la primera, segunda, cuarta y duodécima semana. Comprobando que inicialmente la fuerza de fijación de esta malla fue equivalente a la del TVT tradicional, y aumentó en las diferentes mediciones a través del tiempo. Además se comprobó que sólo una fuerza mayor a $5 \mathrm{~N}$ (500gm) es capas de deformar la cinta. Lo que equivale a una fuerza 10 veces mayor que la tensión media soportada por la fascia en forma normal ${ }^{20}$.En este estudio también se comprobó histológicamente la buena biointegración de la malla.

Durante las últimas dos décadas hemos asistido al nacimiento de un gran número de técnicas quirúrgicas en Uroginecología ${ }^{21}$.Esto ha sido principalmente gracias a la mejor comprensión de la anatomía y fisiología del suelo pélvico, y a la aparición de mallas fabricadas con las características apropiadas $^{22,23}$. La malla del sistema TVT-Secur es de polipropileno, macroporo y monofilamento. En síntesis, se trata de una malla sintética Tipo I, que probadamente ha tenido éxito en las cirugías vaginales ${ }^{24}$, ya que permite una mejor biointegración. Al tener poros mayores de 75 micrones, facilitará el paso de leucocitos, angiogénesis e infiltración de colágeno, proporcionando un mejor anclaje y biointegración. La facilitación en el pasaje de leucocitos y macrófagos (50 micrones), reduce el riesgo de infección (bacterias 1 micrón). El tipo de fibra también puede facilitar la infección. Las mallas multifilamentos, con separación entre ellas menores a 10 micrones, permitirán el paso de bacterias y no de leucocitos. Otra característica favorable de la malla en este sistema, es su tecnología de confección y corte con láser, lo que evita que sus fibras se separen, manteniendo la forma una vez puesta bajo la uretra.

Debemos recordar que los resultados de un sistema de corrección de la incontinencia de orina, no van a depender exclusivamente de la técnica quirúrgica, sino también del tipo y características de la malla utilizada 23,25 . Cualquier malla bajo la uretra no da lo mismo, incluso no sólo puede ser causa de fracaso del sistema, también puede provocar complicaciones.

Esta malla en ambos extremos posee un sistema de fijación, que encierra la malla de polipropileno a manera de un emparedado (sándwich). Este sistema de fijación está formado por Vicryl (poliglactin 910) y PDS (polidioxanon), otorgando un sistema de anclaje responsable de evitar el desplazamiento de la cinta mientras se logra el adicional entregado por la biointegración de la malla.

En resumen la biointegración de la malla sumado al sistema de fijación de ambos extremos, otorga una fuerza de fijación mayor a la que logra la malla por sí sola.

Respecto a la decisión de cuándo utilizar el TVTSecur, para poner la cinta sub-medio-uretral en "U" o en "V". Nuestro grupo de trabajo a optado por utilizarla en "V" cuando se trata de una paciente con incontinencia de esfuerzo pura tipo II. En cambio, cuando se trata de una tipo II asociada a algún grado de deficiencia intrínseca de esfínter (tipo III), utilizamos la posición en "U". Experiencia tabulada y registrada con la técnica clásica, sin publicar aún, en que hemos obtenido muy buenos resultados. En 1999 Kayigil et al. ${ }^{26}$, demostraron en un grupo de 50 pacientes, que el $28 \%$ eran portadoras de incontinencia de orina tipo II asociada a deficiencia 
intrínseca de esfínter. Un estudio actualmente en editorial, realizado por nuestro grupo, de urodinamias en 100 pacientes ingresados para estudio por incontinencia de orina clínica, demostró esta asociación en un 30\%. Diferentes trabajos han demostrado la alta efectividad del TVT (“U”) en mujeres con incontinencia de orina de esfuerzo, hipermovilidad uretral y deficiencia intrínseca de esfínter ${ }^{27}$.

Una experiencia publicada por Moore et al. demostró casos de falla del sistema TOT, tratados exitosamente con un TVT posteriormente, sin remover la cinta transobturadora ${ }^{28}$. En aquella serie de cinco casos, el estudio urodinámico demostró la presencia de deficiencia intrínseca de esfinter asociada a movilidad uretral mayor a 30 grados.

La primera y única experiencia publicada durante este año con TVT-Secur, fue la de Martan et $\mathrm{al}^{12}$. Incluyó 10 pacientes con cinta en "V" y 5 en "U". Completando un seguimiento entre uno y tres meses, con un rango de curación del 93\%. Estos resultados son similares a los publicados con las técnicas de cintas clásicas retropúbicas y transobturadoras, en sintesis se trata de porcentajes exitosos. Aún cuando se trata al igual que nuestra experiencia, del informe preliminar, es importante el seguimiento y medición de los resultados a largo plazo. Sin embargo, al menos teóricamente, permiten suponer que también mantendrán resultados similares a las técnicas clásicas a largo plazo. Respecto a las complicaciones, no se presentaron en los casos de cinta en "U". A diferencia de los casos en "V", en que se presentó un caso de fracaso al persistir la incontinencia, la cinta se dobló en dos casos, una paciente presentó dolor vaginal, y en otro caso se presentó una erosión vaginal de $3 \mathrm{~mm}$. A pesar de que se trata de complicaciones menores y de fácil solución (a excepción del fracaso), aparecen como importantes en número relativo para un grupo total de sólo 15 pacientes. Estas pueden ser parte de la curva de aprendizaje de la técnica, por lo que seguramente se podrán evitar en el futuro. En nuestros pacientes, antes de empezar la experiencia, ensayamos teóricamente y en forma práctica las posibles complicaciones, y tomamos todas las precauciones para evitarlas. Por ejemplo, como ya es parte de nuestros protocolos, en el caso de encontrarse previamente a la cirugía, una mucosa vaginal atrófica, recuperamos su calidad con la utilización de estriol local. Hemos visto que esta precaución reduce la posibilidad de infecciones, de erosiones y mejora la cicatrización. También mantenemos esta indicación durante el período de observación y control postoperatorio.

La presente comunicación es la experiencia inicial, y sólo la incorporación de nuevos pacientes y el seguimiento a largo plazo, permitirán determinar el mantenimiento de los buenos resultados en el tiempo. Al igual como lo hemos hecho con las técnicas antecesoras, TVT y TVT-O ${ }^{17}$, a futuro compararemos las tres técnicas.

\section{CONCLUSIONES}

Basados en nuestra experiencia preliminar, el sistema TVT-Secur es un sistema seguro y eficaz en la corrección de la IOE.

Esta experiencia preliminar, con altos porcentajes de curación iniciales y sin complicaciones, permite suponer resultados promisorios a largo plazo, semejantes los sistemas antecesores. Sin embargo, solo el seguimiento a largo plazo, permitirá confirmar el mantenimiento de estos resultados a largo plazo.

Al tratarse de una técnica de menor invasión que sus antecesores, debiera tener una menor posibilidad de complicaciones, transformándose en una técnica más segura.

\section{REFERENCIAS}

1. MacLennan AH, Taylor AW, Wilson DH, Wilson D. The prevalence of pelvic floor disorders and their relationship to gender, age, parity and mode of delivery. BJOG. 2000;107(12):1460-1470.

2. Hannestad YS, Rortveit G, Sandvik H, Hunskaar S; Norwegian EPICONNT study. Epidemiology of Incontinence in the Country of Nord-Trondelag. A community-based epidemiological survey of female urinary incontinence: the Norwegian EPICONT study. Epidemiology of Incontinence in the Country of Nord-Trondelag. J Clin Epidemiol. 2000;53(11):1150-1157.

3. Ulmsten U, Henriksson L, Johnson P, Varhos G. An ambulatory surgical procedure under local anesthesia for treatment of female urinary incontinence. Int Urogynecol J Pelvic Floor Dysfunct. 1996;7(2):81-85

4. Rezapour M, Ulmsten U. Tension-free vaginal tape (TVT) in women with mixed urinary incontinence -a long-term follow-up. Int J Urogynecol J Pelvic Floor Dysfunct. 2001;12 suppl:15-18.

5. Nilsson GC, Rezapour M, Falconer C. 7 year follow-up on the Tension-free Vaginal Tape (TVT) procedure; International Urogynecology Journal, IUGA Abstract 116 (89): October 2003.

6. Sola Dalenz V, Pardo Schanz J, Ricci Arriola P, Guiloff Fische E, Chiang Miranda H. TVT associated with other gynecological operations in the same procedure: results and complications. Arch Esp Urol. 2005;58(10):983-988.

7. Delorme E. Transobturator urethral suspension: mini-invasive procedure in the treatment of stress urinary incontinence in women. Prog Urol. 2001;11(6):1306-1313. 
8. Delorme E, Droupy S, De Tayrac R, Delmas V. Transobturator tape (Uratape). A new minimally invasive method in the treatment of urinary incontinence in women. Prog Urol. 2003;13 (4):656-659.

9. Pardo Schanz J, Ricci Arriola P, Sola Dalenz V, Tacla Fernández X. Trans-obturator-tape (TOT) for the surgical repair of stress urinary incontinence: our experience. Arch Esp Urol. 2006;59(3):225-232.

10. De Leval J. Novel surgical technique for the treatment of female stress urinary incontinence: transobturator vaginal tape inside-out. Eur Urol. 2003;44(6):724-730.

11. Sola Dalenz V, Pardo Schanz J, Ricci Arriola P, Guiloff Fische E, Chiang Miranda H. Minimal invasive surgery in female urinary incontinence: TVT-O. Actas Urol Esp. 2006;30(1):61-66.

12. Martan A, Masata J, Svabik K TVT SECUR System--tensionfree support of the urethra in women suffering from stress urinary incontinence--technique and initial experience. Ceska Gynekol. 2007 ;72(1):42-49.

13. Slack M, Culligan P, Tracey M, Hunsicker K, Patel B, Sumeray M. Relationship of urethral retro-resistance pressure to urodynamic measurements and incontinence severity. Neurourol Urodyn. 2004;23(2):109-114

14. Slack M, Tracey M, Hunsicker K, Godwin A, Patel B, Sumeray M. Urethral retro-resisteance pressure: a new clinical measure of urethral function. Neurol Urodyn. 2004;23(7):656-661.

15. McGuire EJ, Fitzpatrick CC, Wan J, Bloom D, Sanvordenker J, Ritchey M, Gormley EA. Clinical assessment of urethral sphincter function. J Urol 1993;150 (5 Pt 1): 1452-1454.

16. Abouassaly R, Steinberg JR, Lemieux M, Marois C, Gilchrist LI, Bourque JL, et al. Complications of tension-free vaginal tape surgery: a multi-institutional review. BJU Int. 2004;940:110-113.

17. Sola V, Pardo J, Ricci P, Guiloff E, Chiang H. TVT versus TVT-O for Minimally Invasive Surgical Correction of Stress Urinary Incontinence. Int Braz J Urol. 2007;33(2):246-253; discussion 254.

18. Boyles SH, Edwards R, Gregory W, Clark A. Complications associated with transobturator sling procedures. Int Urogynecol J Pelvic Floor Dysfunct. 2007 Jan; 18(1):19-22.

19. Rezapour M, Novara G, Meier PA, Holste J, Landgrebe S, Artibani W. A 3-month preclinical trial to assess the performance of a new TVT-like mesh (TVTx) in a sheep model. Int Urogynecol J Pelvic Floor Dysfunct. 2007;18(2):183-187.
20. Lin AT, Wang SJ, Chen KK, Chang LS. In vivo tension sustained by fascial sling in pubovaginal sling surgery for female stress urinary incontinence. J Urol. 2005;173(3):894-897.

21. Martan A. New options in reconstructive pelvic floor surgery and surgery in urogynecology. Ceska Gynekol. 2006;71(6):455463.

22. Sola V, Pardo J, Ricci P, Guiloff E. Tension free monofilament macropore polypropylene mesh (Gynemesh PS) in female genital prolapse repair. Int Braz J Urol. 2006 Jul-Aug;32(4):410-4; discussion 415 .

23. Sola Dalenz V, Pardo Schanz J, Ricci Arriola P, Guiloff Fische E.Tension free monofilament polypropylene mesh in cystocele and rectocele concomitant repair. Actas Urol Esp. 2005;29 (10): 977-980.

24. Roth CC, Holley TD, Winters JC. Synthetic sling: which material, which approach. Curr Opin Urol. 2006 Jul; 16(4):234-239

25. Galmes Belmonte I, Diaz Gomez E. The devices used to correct the urinary incontinence by tension-free meshes. Are all them equals?. Actas Urol Esp. 2004;28(7): 487-496.

26. Kayigil O, Iftekhar Ahmed S, Metin A. The coexistence of intrinsic sphincter deficiency with type II stress incontinence. J Urol. 1999;162(4):1365-1366.

27. Meschia M, Pifarotti P, Buonaguidi A, Gattei U, Spennacchio M. Tension-free vaginal tape (TVT) for treatment of stress urinary incontinence in women with low-pressure urethra. Eur J Obstet Gynecol Reprod Biol. 2005;122(1):118-121.

28. Moore RD, Gamble K, Miklos JR. Tension-free vaginal tape sling for recurrent stress incontinence after transobturator tape sling failure. Int Urogynecol L Pelvic Floor Dysfunct. 2007; 18(3):309-313.

Correspondencia autor: Dr. P. Ricci Arriola

Unidad de Uroginecología y Cirugía Vaginal

Clínica Las Condes

Lo Fontecilla 441. Las Condes, Santiago (Chile)

Tel.: (56) 22104610

E-mail autor: pricci@clinicalascondes.cl

Información artículo: Original - Incontinencia urinaria

Trabajo recibido: junio 2007

Trabajo aceptado: noviembre 2007 